\title{
Kardinalitätserhöhende Vervollständigungen metrischer und normierter Räume
}

Von

\section{Gerald Kuba}

(Vorgelegt in der Sitzung der math.-nat. Klasse am 10. April 2003 durch das w. M. Edmund Hlawka)

Es sei $X$ ein beliebiger metrischer Raum und $\widetilde{X}$ seine Vervollständigung. Die klassische Vervollständigung von $\mathbb{Q}$ demonstriert, daß $|\widetilde{X}|>|X|$ möglich ist. Es kann also mit der Vervollständigung eines metrischen Raumes seine Kardinalität vergrößert werden. Das ist allerdings nicht möglich, wenn der Ausgangsraum $X$ von der Mächtigkeit des Kontinuums ist, da die Anzahl der Äquivalenzklassen von Cauchyfolgen in $X$ trivialerweise durch die Mächtigkeit des gesamten Folgenraumes $X^{\mathbb{N}}$ beschränkt ist und im Falle $|X|=2^{\aleph_{0}}$ eben $\left|X^{\mathbb{N}}\right|=|X|$ gilt. Eine Kardinalitätssteigerung durch Vervollständigung ist daher speziell bei allen gängigen normierten Räumen (über $\mathbb{R}$ oder $\mathbb{C}$ ) unmöglich. (Auch bei endlichen metrischen Räumen ist dies unmöglich, sind dieselben doch ohnehin bereits vollständig.) Da einem ferner im Alltag an metrischen Räumen, die mächtiger als das Kontinuum sind, immer nur vollständige (entweder diskrete oder Banachräume) über den Weg laufen, ist es naheliegend zu fragen, ob es überhaupt normierte bzw. überabzählbare metrische Räume $X$ gibt, für die $|\widetilde{X}|>|X|$ gilt. Tatsächlich kann diese Fragestellung, die in der Literatur nicht thematisiert bzw. (wie in [2]) nur rudimentär angegangen wird, im Rahmen einer kurzen Note elementar abgehandelt und restlos erledigt werden. Die folgenden 
Resultate sind daher als kleine Ergänzungen des Handbuchs [2] anzusehen, wo lediglich (vgl. [2] Theorem 8.3) $|\widetilde{X}| \in\left\{\kappa, \kappa^{\aleph_{0}}\right\}$ für die Dichte $\kappa$ eines vollständigen Raumes $\widetilde{X}$ gezeigt wird.

Satz 1. Es gibt beliebig große normierte Räume, deren Kardinalität sich durch Vervollständigung steigern läßt. Genauer läßt sich zu einer Kardinalzahl $\kappa$ mit $\kappa \geq 2^{\aleph_{0}}$ dann und nur dann ein normierter Raum $X$ mit $\kappa=|X|<|\widetilde{X}|$ angeben, wenn $\kappa^{\aleph_{0}}>\kappa$ gilt.

Satz 2. Unter Annahme der Kontinuumshypothese $2^{\aleph_{0}}=\aleph_{1}$ hat der kleinste metrische Raum $X$ mit $|\widetilde{X}|>|X|>\aleph_{0}$ die Kardinalität $\aleph_{\omega}$.

Satz 3. Unter der Annahme, daß die Kontinuumshypothese falsch ist, hat der kleinste überabzählbare metrische Raum X mit $|\widetilde{X}|>|X|$ die Kardinalität $\aleph_{1}$. Darüber hinaus gibt es zu jeder transfiniten Kardinalzahl $\lambda<2^{\aleph_{0}}$ einen metrischen (aber natürlich keinen normierten) Raum $X$ mit $\lambda=|X|<|\widetilde{X}|$.

Beweis von Satz 1. Im Falle $\kappa^{\aleph_{0}}=\kappa$ ist $\kappa=|X|<|\widetilde{X}|$ wegen $\left|X^{\mathbb{N}}\right|=|X|$ unmöglich. Es sei also $\kappa \geq 2^{\aleph_{0}}$ irgendeine Kardinalzahl, für die $\kappa^{\aleph_{0}}>\kappa$ gilt. Natürlich gibt es eine Unmenge solcher Kardinalzahlen, z.B. alle der Art $\aleph_{\alpha+\omega}>2^{\aleph_{0}}$ (vgl. [1]), sodaß wir fertig sind, wenn wir einen normierten Raum $X$ mit $|X|=\kappa$ und $|\widetilde{X}|=\kappa^{\aleph_{0}}$ angeben. Wir zeigen nun etwas mehr, indem wir gleich einen Hilbertraum $\widetilde{X}$ der Kardinalität $\kappa^{\aleph_{0}}$ konstruieren, der einen unvollständigen, dichten Innenproduktraum $X$ der Kardinalität $\kappa$ enthält. Bei der Konstruktion dieser Räume ist der Spielraum sehr eng, da bekanntlich jeder Hilbertraum normisomorph zu einem $l^{2}(\Lambda)$ ist. Für eine unendliche Indexmenge $\Lambda$ und einen Unterkörper $\mathbb{K}$ von $\mathbb{C}$ sei $\mathcal{A}(\Lambda, \mathbb{K})$ bzw. $\mathcal{E}(\Lambda, \mathbb{K})$ die Menge aller Funktionen $x: \Lambda \rightarrow \mathbb{K}$, für die $x(\lambda) \neq 0$ für höchstens abzählbar bzw. endlich viele $\lambda \in \Lambda$ gilt. Da $\mathcal{A}(\Lambda, \mathbb{K})$ bzw. $\mathcal{E}(\Lambda, \mathbb{K})$ im wesentlichen durch

$$
\bigcup\left\{\mathbb{K}^{A}|A \subset \Lambda \wedge| A \mid=\aleph_{0}\right\} \quad \text { bzw. } \bigcup\left\{\mathbb{K}^{E}|E \subset \Lambda \wedge| E \mid<\aleph_{0}\right\}
$$

gegeben ist und $\left|\left\{A \subset \Lambda|| A \mid=\aleph_{0}\right\}\right|=|\Lambda|^{\aleph_{0}}$ und $\mid\{E \subset \Lambda|| E \mid<$ $\left.\aleph_{0}\right\}|=| \Lambda \mid$ gilt, bekommen wir $|\mathcal{E}(\Lambda, \mathbb{K})|=|\Lambda||\mathbb{K}|$, sowie $|\mathcal{A}(\Lambda, \mathbb{K})|=|\Lambda|^{\aleph_{0}}|\mathbb{K}|^{\aleph_{0}}=|\Lambda|^{\aleph_{0}} \cdot 2^{\aleph_{0}}=|\Lambda|^{\aleph_{0}}$.

Mit $\mathbb{K}=\mathbb{R}$ setzen wir nun standardgemäß $l^{2}(\Lambda):=\{x \in \mathcal{A}(\Lambda, \mathbb{R}) \mid$ $\left.\sum_{\lambda \in \Lambda} x(\lambda)^{2}<\infty\right\}$. Mit dem kanonischen reellen Innenprodukt $x_{1} \cdot x_{2}:=\sum_{\lambda \in \Lambda} x_{1}(\lambda) x_{2}(\lambda)$ und der davon induzierten Metrik $d\left(x_{1}, x_{2}\right)=\sqrt{\sum_{\lambda \in \Lambda}\left(x_{1}(\lambda)-x_{2}(\lambda)\right)^{2}}$ ist dann $l^{2}(\Lambda)$ das klassische 
Modell eines (reellen) Hilbertraums. Natürlich gilt $\left|l^{2}(\Lambda)\right|=$ $|\mathcal{A}(\Lambda, \mathbb{R})|=|\Lambda|^{\aleph_{0}}$. Tatsächlich enthält ja $l^{2}(\Lambda)$ jedenfalls die Menge

$$
\begin{aligned}
\mathcal{F}(\Lambda) & :=\left\{x \in \mathbb{R}^{\Lambda} \mid \exists \varphi \in \mathcal{I}(\Lambda): x(\Lambda \backslash \varphi(\mathbb{N}))\right. \\
& \left.=\{0\} \wedge(x(\varphi(n)))_{n \in \mathbb{N}} \in \prod_{n=1}^{\infty}\left[0, \frac{1}{n}\right]\right\},
\end{aligned}
$$

wobei $\mathcal{I}(\Lambda)$ die Menge aller injektiven Abbildungen von $\mathbb{N}$ nach $\Lambda$ ist, sodaß $|\mathcal{F}(\Lambda)|=|\Lambda|^{\aleph_{0}}$ wegen $|\mathcal{I}(\Lambda)|=|\Lambda|^{\aleph_{0}}$ und $\left|\prod\left[0, \frac{1}{n}\right]\right|=2^{\aleph_{0}}$ gilt. Ferner ist $\mathcal{E}(\Lambda, \mathbb{R})$ offensichtlich ein dichter Teilraum von $l^{2}(\Lambda)$, dessen Kardinalität durch $|\mathcal{E}(\Lambda, \mathbb{R})|=|\Lambda| \cdot 2^{\aleph_{0}}$ gegeben ist. Mit der Festlegung auf irgendeine Indexmenge $\Lambda$ der Kardinalität $\kappa$ hat man schließlich mit $X=\mathcal{E}(\Lambda, \mathbb{R})$ einen normierten Raum der Kardinalität $\kappa$ gewonnen, dessen Vervollständigung $\widetilde{X}=l^{2}(\Lambda)$ die größere Mächtigkeit $\kappa^{\aleph_{0}}$ besitzt. Will man die Geschichte lieber im Komplexen durchspielen, so braucht man im Beweis nur $\mathbb{R}$ durch $\mathbb{C}$ zu ersetzen und die entsprechenden Adaptationen vorzunehmen.

Beweis von Satz 2. Die Voraussetzung $2^{\aleph_{0}}=\aleph_{1}$ erzwingt $\aleph_{n}^{\aleph_{0}}=\aleph_{n}$ für $0<n<\omega$ nach der HAUSDORFFschen Formel $\aleph_{\alpha+1}^{\kappa}=\aleph_{\alpha}^{\kappa} \cdot \aleph_{\alpha+1}$ und verhindert daher die Existenz eines metrischen Raumes $X$ mit $\aleph_{0}<|X|<\aleph_{\omega}$ und $|\widetilde{X}|>|X|$. Wegen $\aleph_{\omega}^{\aleph_{0}}>\aleph_{\omega}$ folgt somit Satz 2 sofort aus Satz 1.

Beweis von Satz 3. In Anlehnung an den Beweis von Satz 1 sei nun $X=\mathcal{E}(\Lambda, \mathbb{Q})$ mit derselben Metrik wie vorher versehen. ( $X$ ist im strengen Sinn kein normierter Raum. Wenn man will, kann man ihn natürlich als normierten Raum über $\mathbb{Q}$ mit Normwerten in $\mathbb{R}$ ansehen.) Mit geeigneten approximierenden Folgen erkennt man leicht, da $\beta X$ ein dichter Teilraum des vollständigen metrischen Raumes $l^{2}(\Lambda)$ ist, sodaß also $\widetilde{X}=l^{2}(\Lambda)$ gilt. Mit einer beliebig gewählten unendlichen Indexmenge $\Lambda$ der Kardinalität $\lambda<2^{\aleph_{0}}$ sind wir daher auch schon fertig, da einerseits $|X|=|\mathcal{E}(\Lambda, \mathbb{Q})|=|\Lambda||\mathbb{Q}|=\lambda \cdot \aleph_{0}=\lambda$, andererseits $|\widetilde{X}|=\left|l^{2}(\Lambda)\right|=|\Lambda|^{\aleph_{0}}=\lambda^{\aleph_{0}}=2^{\aleph_{0}}$ gilt.

Abschließend wollen wir noch klären, ob das Herausfaktorisieren der Cauchyfolgen aus dem Folgenraum $X^{\mathbb{N}}$ auf nichttriviale Weise kardinalitätsmindernd sein kann.

Satz 4. Es gibt keinen metrischen Raum X dergestalt, daß $|X|<|\widetilde{X}|<$ $\left|X^{\mathbb{N}}\right|$ gilt.

Beweis. Es ist klar, daß $X$ nicht endlich sein kann. Nach [2] Theorem 8.3 gilt für die Dichte $\kappa$ von $\widetilde{X}$ entweder $\kappa=|\widetilde{X}|$ oder $\kappa^{\aleph_{0}}=|\widetilde{X}|$. Im 
Falle $|X|<|\widetilde{X}|$ gilt somit $|\widetilde{X}|=\kappa^{\aleph_{0}}$ wegen $\kappa \leq|X|$. Dann bekommen wir aber $\left|X^{\mathbb{N}}\right| \leq\left|\widetilde{X}^{\mathbb{N}}\right|=\left(\kappa^{\aleph_{0}}\right)^{\aleph_{0}}=\kappa^{\aleph_{0}}=|\widetilde{X}|$.

\section{Literatur}

[1] JeCH, T. (1978) Set Theory. Academic Press, New York

[2] Kunen/Vaughan (eds.) (1984) Handbook of Set-Theoretic Topology. NorthHolland, Amsterdam

Anschrift des Verfassers: ao. Univ.-Prof. Mag. Dr. GERALD KUBA, Institut für Mathematik, Universität für Bodenkultur, Gregor Mendel-Straße 33, 1180 Wien, Österreich. 